\title{
Ultrasound elastography as a potential method to evaluate entrapment neuropathies in elite athletes: a mini-review
}

\author{
Agnieszka Dąbrowska-Thing ${ }^{\mathrm{E}, \mathrm{F}}$, Jakub Zakrzewski ${ }^{\mathrm{F}}$, Oleg Nowak ${ }^{\mathrm{F}}$, Żaneta Nitek ${ }^{\mathrm{F}}$ \\ Gruca Orthopaedic and Trauma Teaching Hospital, Centre of Postgraduate Medical Education, Otwock, Poland
}

\section{Abstract}

\begin{abstract}
Entrapment neuropathies constitute a significant albeit still neglected problem in athletes. Currently available diagnostic options in patients with suspected neuropathies, nerve conduction studies, and magnetic resonance imaging have some drawbacks, mostly related to their invasive character, high cost, and limited availability. This paper reviews published articles documenting the use of ultrasound elastography, a novel technique to determine tissue strain, in the evaluation of nerve stiffness in entrapment neuropathy, and it discusses potential pros and cons of this method in the examination of elite athletes. Based on the review of literature, ultrasound elastography - especially shear wave elastography - seems suitable for the evaluation of entrapment neuropathies in elite athletes. Published evidence from the general population suggests that most common entrapment neuropathies (carpal tunnel syndrome, ulnar neuropathy of the elbow, ulnar tunnel syndrome) are associated with a significant increase in the stiffness of affected nerves, which can be accurately detected with ultrasound elastography. Before adding ultrasound elastography to the armamentarium of diagnostic tests used routinely in athletes, its accuracy in the detection of entrapment neuropathies needs to be verified in this specific group, and the reference values for peripheral nerve strain in sportspersons need to be determined.
\end{abstract}

Key words: elastography, neuropathy, athletics, ultrasound (US).

\section{Introduction}

Entrapment neuropathies are a common cause of pain and motor dysfunction [1]. The neuropathy is typically a consequence of an increase in intraneural pressure, which leads to nerve oedema, impairment of blood perfusion, ischaemia, and eventually demyelination, axonal atrophy, and secondary fibrosis [2]. Entrapment neuropathies constitute a significant, albeit still neglected, problem in athletes. The aetiology of nerve pathologies in sportspersons varies and may include an injury (blunt trauma or excessive stretching), instability, or compression; neuropathy may also be a consequence of incorrect biomechanics, use of inappropriate equipment, wrong technique, or training errors. Practicing sports may cause enforced and repeated compression, stretching, tearing or subluxation of peripheral nerves [3]. The exact incidence of entrapment neuropathies in athletes is unknown, but it is estimated that in approximately $80 \%$ of cases nerves of the upper extremity are affected [4].

Morphological and functional changes within the nerve usually precede clinical manifestation of entrapment neuropathy, which highlights the importance of diagnostic methods capable of detecting this condition at its earliest, subclinical stages. Currently, the gold standard in early diagnosis of most entrapment neuropathies is via nerve conduction studies (NCS). Some morphological changes within peripheral nerves can also be detected on magnetic resonance imaging (MRI). However, both these methods have their drawbacks, mostly related to their invasive character, high cost, and limited availability $[5,6]$. Progress in ultrasound techniques stimulated interest in this method

\section{Correspondence address:}

Dr. Agnieszka Dąbrowska-Thing, Gruca Orthopaedic and Trauma Teaching Hospital, Centre of Postgraduate Medical Education, 13 Konarskiego St.,

05-400 0twock, Poland, e-mail: aga.dabrowska@poczta.fm

Authors' contribution:

A Study design · B Data collection · C Statistical analysis · D Data interpretation · E Manuscript preparation · F Literature search · G Funds collection 


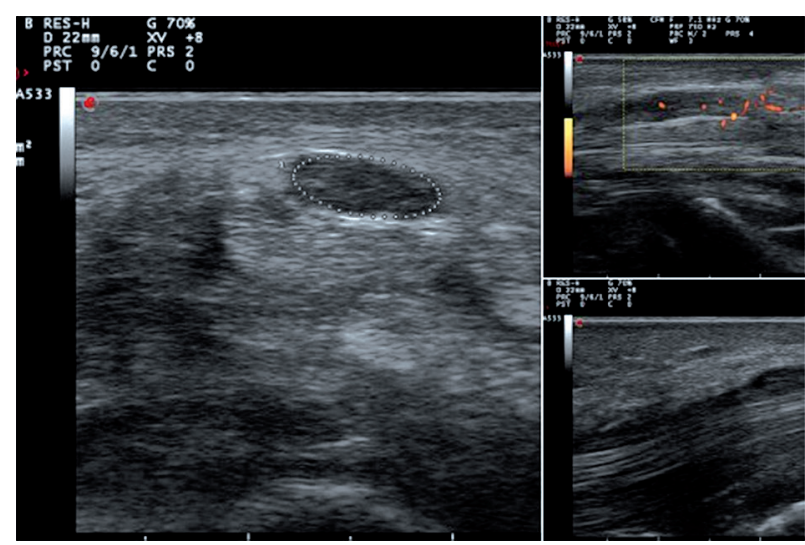

Figure 1. Median nerve neuropathy within the carpal tunnel: increased cross-sectional area of the nerve $\left(16 \mathrm{~mm}^{2}\right)$, loss of fascicular structure, enhanced blood perfusion

as a diagnostic modality in patients with suspected entrapment neuropathies. Indeed, conventional grey-scale ultrasound proved to be accurate in the detection of structural abnormalities within entrapped peripheral nerves, and ultrasonographic measurement of nerve cross-sectional area (CSA) at the entrapment site became an accessory diagnostic test in this indication (Figure 1) [7,8]. However, although many studies demonstrated that entrapment neuropathy is associated with a significant increase in the CSA of the affected nerves, this parameter was also shown to suffer from a lack of standardisation, which negatively affected its sensitivity and specificity $[8,9]$.

A novel alternative to conventional ultrasound in the evaluation of peripheral nerves can be ultrasound elastography, an imaging method dedicated to the determination of tissue strain. Currently available elastographic techniques can be classified into static and dynamic ones. Static elastography, most often in the form of strain elastography (SE), is a semiquantitative technique providing information about the relative strain of tissues. The strain of examined tissue or organ is colour-coded and compared with a reference scale. In contrast to SE, dynamic elastography, usually shear wave elastography (SWE), measures absolute stiffness of tissues, which is expressed as shear wave velocity (in metres per second, $\mathrm{m} / \mathrm{s}$ ) or converted to Young modulus (in kilopascals, $\mathrm{kPa}$ ). Due to its simplicity, non-invasive character, and high accuracy, ultrasound elastography has already been recognised by specialists in many medical disciplines, such as oncology, hepatology [10], dermatology [11-13], and angiology [14,15].

Considering the pathomechanism of entrapment neuropathy, with progressive oedema and fibrosis, both of which are likely to be associated with changes in nerve stiffness, ultrasound elastography seems to be applicable also for the evaluation of patients with this condition, among them elite athletes. In this paper, we review published articles documenting the use of ultrasound elastography in this indication and discuss the potential pros and cons of this method in the examination of elite athletes.

\section{Material and methods}

A systematic search of the PubMed, Scopus, and Google Scholar was carried out. All published studies that examined the usefulness of ultrasound elastography in the evaluation of peripheral nerves and used the terms 'elastography' OR 'ultrasound elastography' OR 'sonoelastography' OR 'strain elastography' OR 'shear wave elastography' AND 'neuropathy' OR 'peripheral neuropathy' OR 'entrapment neuropathy' OR 'nerve entrapment' OR 'carpal tunnel syndrome' OR 'pronator teres syndrome' OR 'anterior interosseus syndrome' OR 'ulnar neuropathy of the elbow' OR 'ulnar tunnel syndrome' OR 'radial neuropathy' OR 'supinator syndrome' were identified and reviewed. The search limits were defined as 'English' (language), and 'the beginning of a given database through to 31 July 2019 ' (publication date).

\section{Carpal tunnel syndrome}

Carpal tunnel syndrome (CTS) results from compression of the median nerve within the carpal tunnel. The direct cause of the neuropathy is impaired perfusion of the nerve associated with an increase in carpal tunnel pressure, caused by a trauma or monotypic movements with hyperextension of the wrist. Because of the latter mechanism, CTS is particularly common in cyclists, bowlers, bodybuilders, wrestlers, and wheelchair athletes $[16,17]$.

Although currently the gold standard in the evaluation of CTS is NCS, a few published studies analysed the applicability of ultrasound elastography in this indication. The early studies with semiquantitative SE demonstrated that CTS is associated with a significant increase in median nerve stiffness within the carpal tunnel $[18,19]$. These findings were later confirmed in SWE-based studies that included a total of 219 wrists from patients with CTS and 116 wrists of healthy controls [20-22]. Median values of Young modulus for median nerves in patients with CTS ranged between 66.7 and $100 \mathrm{kPa}$ and were significantly higher than the stiffness of the median nerve in healthy controls (32-42 kPa). Importantly, those studies documented also a considerable variance in SWE stiffness of median nerve, whether in patients with CTS or in healthy controls. Hence, it is still unclear which cut-off value of median nerve stiffness could be used to accurately distinguish between patients with CTS and without. In the study conducted by Kantarci et al. [20], a cut-off value of $40.4 \mathrm{kPa}$ provided an excellent diagnostic accuracy in the detection of CTA. However, Paluch et al. [22] identified a cut-off value nearly twice as high $(79 \mathrm{kPa})$ with equally good parameters of diagnostic accuracy. Considering high individual variance of elastographic parameters, the latter authors suggested that instead of absolute stiffness of median nerve, CTS should be diagnosed based on the wristto-forearm ratio of median nerve stiffness, obtained by dividing median nerve stiffness within the carpal tunnel 
by its stiffness on the forearm. In the study conducted by those authors, the stiffness ratios in CTS patients turned out to be significantly higher than in the controls (2.1 vs. $1.0)$ and provided excellent accuracy in distinguishing between these two groups [22].

While the abovementioned results show clearly that CTS is associated with an increase in median nerve strain within the carpal tunnel, a link between the elastographic parameters and severity of the neuropathy is not straightforward. Although some authors demonstrated that median nerve stiffness increased proportionally to CTS severity [20], other researchers did not find a significant association between these variables [22,23]. Finally, in the SE study conducted by Ghajarzadeh et al. [19], the semiquantitative stiffness of median nerve increased significantly with CTS severity, but not in the most severe form of the disease. The authors interpreted this observation as a consequence of axonal loss at more advanced CTS stages [19].

\section{Other entrapment neuropathies}

Except for CTS, published evidence on the usefulness of ultrasound elastography in patients with other suspected neuropathies occurring commonly in athletes are sparse.

Ulnar neuropathy of the elbow (UNE) is the second most common entrapment neuropathy of the upper extremity [24]. The frequent occurrence of this neuropathy is associated with the superficial location of the ulnar nerve at the elbow, which makes it prone to injury [25]. Not surprisingly, the risk of UNE is particularly high among athletes, especially cyclists, baseball and football players, bodybuilders, and wrestlers [26,27]. We identi- fied only one published study using ultrasound elastography to evaluate ulnar nerve in patients with UNE. Paluch et al. [28] used SWE to determine ulnar nerve strain within the cubital tunnel in 34 patients with UNE and in 38 healthy controls. Mean SWE of the ulnar nerve in patients with UNE turned out to be significantly higher than in the controls (96.38 $\pm 9.62 \mathrm{kPa}$ vs. $33.08 \pm 10.13 \mathrm{kPa}$ ) (Figure 2). Moreover, patients with this neuropathy presented with significantly higher values of ulnar nerve stiffness ratio in the cubital tunnel to its stiffness at the distal arm (2.8 vs. 1.0$)$ and mid-arm (2.7 vs. 1.0$)$. A cut-off value of ulnar nerve stiffness of $\geq 61 \mathrm{kPa}$ and ulnar nerve stiffness ratios at distal arm and mid-arm of $\geq 1.68$ and $\geq 1.75$, respectively, provided an excellent accuracy in distinguishing between patients with UNE and without [28].

Ulnar tunnel syndrome (UTS) is associated with compression of ulnar nerve within Guyon's canal at the wrist level [29]. Among athletes, ulnar tunnel syndrome occurs more often in long-distance cyclists [27] but was also described in wheelchair athletes, cross-country skiers, weightlifters, and tennis, baseball, and golf players [17]. To the best of our knowledge, the applicability of ultrasound elastography for the evaluation of ulnar nerve in UTS was the subject of only one published study. Paluch et al. [30] used SWE to examine the ulnar nerve in 46 patients with established UTS and in 39 healthy controls. Patients with UTS presented with significantly higher mean stiffness of the nerve within Guyon's canal than the controls $(99.41 \mathrm{kPa}$ vs. $49.08 \mathrm{kPa}$ ). Both ulnar nerve stiffness of $\geq 80 \mathrm{kPa}$ and the ratios of ulnar nerve stiffness at Guyon's canal to its stiffness at the distal or proximal forearm of $\geq 1.5$ provided excellent, $100 \%$ accuracy in distinguishing between patients with UTS and without [30].
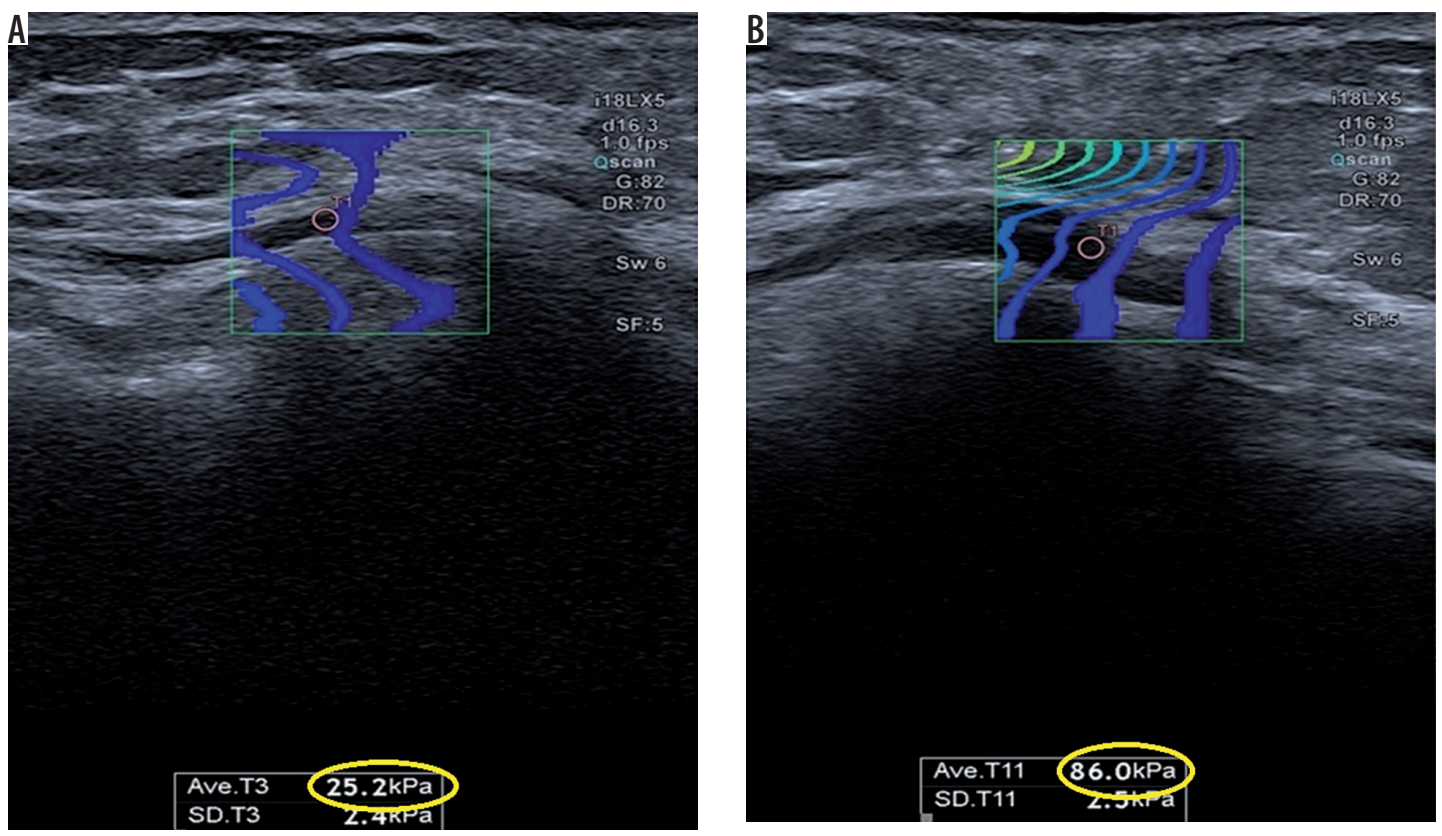

Figure 2. Shear wave elastography (SWE) of the ulnar nerve within the cubital tunnel. A) Normal nerve, low SWE strain. B) Ulnar neuropathy, high SWE strain 


\section{Pros and cons of ultrasound elastography in the evaluation of entrapment neuropathies in elite athletes}

Based on the data reviewed above, an increase in peripheral nerve stiffness detected using ultrasound elastography, in particular, SWE might be a diagnostic marker of entrapment neuropathy. Furthermore, sparse available evidence suggests that elastographic measurements might be highly reproducible, with inter-rater agreement rates for SWE median nerve stiffness in CTS patients and healthy controls of 0.848 and 0.809 , respectively [20]. These arguments support the routine use of ultrasound elastography in the evaluation of patients with suspected entrapment neuropathies, among them athletes.

However, several issues need to be addressed before adding elastography to the armamentarium of imaging studies used in sports medicine. First, available information on the applicability of ultrasound elastography is limited to a few entrapment neuropathies, primarily CTS; some other conditions from this group diagnosed commonly among sportspersons, e.g. pronator teres syndrome, anterior interosseus syndrome, radial neuropathy, and supinator syndrome, have not been examined with this method thus far. Second, none of the previous studies included elite athletes (or this fact was not reported), and it cannot be excluded that the underlying mechanisms of entrapment neuropathy and dynamics of the pathological process in this group might be different than in the general population. This problem is currently a subject of research carried out by our group among professional cyclists. Third, the use of ultrasound elastography as a diagnostic test should be preceded by the determination of reference values for peripheral nerve stiffness. To the best of our knowledge, determination of median nerve stiffness in healthy persons has been the subject of only two published studies. The results of those studies differed consid- erably, with mean median nerve stiffness at the wrist equal to $20.78 \pm 5.11 \mathrm{kPa}$ [31] and $32.26 \pm 18.6 \mathrm{kPa}$ [32]. Furthermore, it needs to be verified if the values determined in the general population are also applicable to elite athletes. Fourth, as mentioned above, it is still unclear whether peripheral nerve stiffness can be considered a measure of neuropathy severity. Fifth, all of the above-mentioned studies were carried out in patients in whom entrapment neuropathy had already been diagnosed with NCS, and hence we do not know whether elastography could be used to detect this condition at the earliest preclinical stages. Finally, two technical questions are still a matter of debate: whether the SWE stiffness of peripheral nerves can change depending on the plane on which the measurements were taken [33], and if bone surfaces in proximity of peripheral nerves might act as hardening artefacts [32] that impair homogeneous shear wave propagation [34].

\section{Conclusions}

Ultrasound elastography, especially SWE, seems suitable for the evaluation of entrapment neuropathies in elite athletes. Published evidence from the general population suggests that most common entrapment neuropathies are associated with a significant increase in the stiffness of affected nerves, which can be accurately detected with ultrasound elastography. Before adding ultrasound elastography to the armamentarium of diagnostic tests used routinely in athletes, its accuracy in the detection of entrapment neuropathies needs to be verified in this specific group, and the reference values for peripheral nerve strain in sportspersons need to be determined.

\section{Conflict of interest}

The authors declare no conflict of interest.

\section{References}

1. Schon LC. Nerve entrapment, neuropathy, and nerve dysfunction in athletes. Orthop Clin North Am 1994; 25: 47-59.

2. Watson JC, Dyck PJ. Peripheral neuropathy: a practical approach to diagnosis and symptom management. Mayo Clin Proc 2015; 90: 940-951.

3. Peck E, Strakowski JA. Ultrasound evaluation of focal neuropathies in athletes: a clinically-focused review. Br J Sports Med 2015; 49: 166-175.

4. Doughty CT, Bowley MP. Entrapment neuropathies of the upper extremity. Med Clin North Am 2019; 103: 357-370.

5. Andreisek G, Crook DW, Burg D, et al. Peripheral neuropathies of the median, radial, and ulnar nerves: MR imaging features. Radiographics 2006; 26: 1267-1287.

6. Dyck PJ, Overland CJ, Low PA, et al. Signs and symptoms versus nerve conduction studies to diagnose diabetic sensorimotor polyneuropathy: Cl vs. NPhys trial. Muscle Nerve 2010; 42: 157-164.
7. Klauser AS, Halpern EJ, De Zordo T, et al. Carpal tunnel syndrome assessment with US: value of additional cross-sectional area measurements of the median nerve in patients versus healthy volunteers. Radiology 2009; 250: 171-177.

8. Pastare D, Therimadasamy AK, Lee E, et al. Sonography versus nerve conduction studies in patients referred with a clinical diagnosis of carpal tunnel syndrome. J Clin Ultrasound 2009; 37: 389-393.

9. Sernik RA, Abicalaf CA, Pimentel BF, et al. Ultrasound features of carpal tunnel syndrome: a prospective case-control study. Skeletal Radiol 2008; 37: 49-53.

10. Shiina T, Nightingale KR, Palmeri ML, et al. WFUMB guidelines and recommendations for clinical use of ultrasound elastography: Part 1: basic principles and terminology. Ultrasound Med Biol 2015; 41: 1126-1147. 
11. Paluch L, Pietruski P, Kwiek B. Age-related changes in elastographically determined strain of the facial fat compartments: a new frontier of research on face aging processes. Adv Dermatol Allergol 2018; doi: https://doi.org/10.5114/ada.2018.79778.

12. Paluch L, Pietruski P, Noszczyk B, et al. Intra-rater reproducibility of shear wave elastography in the evaluation of facial skin. Adv Dermatol Allergol 2019; doi: https://doi.org/10.5114/ada.2018.81144.

13. Paluch L, Ambroziak M, Pietruski P, et al. Shear wave elastography in the evaluation of facial skin stiffness after focused ultrasound treatment. Dermatol Surg 2019; 45: 1620-1626.

14. Paluch $€$, Nitek Ż, Sklinda K, et al. Application of the probit method for elastographic evaluation of venous obliterating material after sclerotherapy procedure. Pol J Radiol 2019; 84: e9-e15.

15. Paluch $€$, Nitek $\dot{Z}$, Sklinda K, et al. Factors influencing elastographically determined remodeling of venous thrombi. J Ultrasound Med 2019; 38: 2643-2650.

16. Palmer D. Carpal tunnel syndrome in athletes. Oper Tech Sports Med 1996; 4: 33-39.

17. Cass S. Upper extremity nerve entrapment syndromes in sports: an update. Curr Sports Med Rep 2014; 13: 16-21.

18. Orman G, Ozben S, Huseyinoglu N, et al. Ultrasound elastographic evaluation in the diagnosis of carpal tunnel syndrome: initial findings. Ultrasound Med Biol 2013; 39: 1184-1189.

19. Ghajarzadeh M, Dadgostar M, Sarraf P, et al. Application of ultrasound elastography for determining carpal tunnel syndrome severity. Jpn J Radiol 2015; 33: 273-278.

20. Kantarci F, Ustabasioglu FE, Delil S, et al. Median nerve stiffness measurement by shear wave elastography: a potential sonographic method in the diagnosis of carpal tunnel syndrome. Eur Radiol 2014; 24 : 434-440.

21. Zhang C, Li M, Jiang J, et al. Diagnostic value of virtual touch tissue imaging quantification for evaluating median nerve stiffness in carpal tunnel syndrome. J Ultrasound Med 2017; 36: 1783-1791.

22. Paluch $€$, Pietruski $P$, Walecki J, et al. Wrist to forearm ratio as a median nerve shear wave elastography test in carpal tunnel syndrome diagnosis. J Plast Reconstr Aesthet Surg 2018; 71: 1146-1152.
23. Tatar IG, Kurt A, Yavasoglu NG, et al. Carpal tunnel syndrome: elastosonographic strain ratio and cross-sectional area evaluation for the diagnosis and disease severity. Med Ultrason 2016; 18: 305-311.

24. Cutts S. Cubital tunnel syndrome. Postgrad Med J 2007; 83: 28-31.

25. Assmus H, Antoniadis G, Bischoff C, et al. Cubital tunnel syndrome - a review and management guidelines. Cent Eur Neurosurg 2011; 72: 90-98.

26. Toth C, McNeil S, Feasby T. Peripheral nervous system injuries in sport and recreation: a systematic review. Sports Med 2005; 35: $717-$ 738.

27. Brubacher JW, Leversedge FJ. Ulnar neuropathy in cyclists. Hand Clin 2017; 33: 199-205.

28. Paluch $€$, Noszczyk B, Nitek $\dot{Z}$, et al. Shear-wave elastography: a new potential method to diagnose ulnar neuropathy at the elbow. Eur Radiol 2018; 28: 4932-4939.

29. Bachoura A, Jacoby SM. Ulnar tunnel syndrome. Orthop Clin North Am 2012; 43: 467-474.

30. Paluch $€$, Noszczyk BH, Walecki J, et al. Shear-wave elastography in the diagnosis of ulnar tunnel syndrome. J Plast Reconstr Aesthet Surg 2018; 71: 1593-1599.

31. Bedewi MA, Coraci D, Ruggeri F, et al. Shear wave elastography of median nerve at wrist and forearm. Heterogeneity of normative values. J Plast Reconstr Aesthet Surg 2019; 72: 137-171.

32. Bortolotto C, Turpini E, Felisaz P, et al. Median nerve evaluation by shear wave elastosonography: impact of "bone-proximity" hardening artifacts and inter-observer agreement. J Ultrasound 2017; 20: 293-299.

33. Paluch L, Pietruski P, Walecki J, et al. Shear wave elastography of median nerve at wrist and forearm. Heterogeneity of normative values: response to authors. J Plast Reconstr Aesthet Surg 2019; 72: 137-171.

34. Bhatia KS, Cho CC, Tong CS, et al. Shear wave elastography of focal salivary gland lesions: preliminary experience in a routine head and neck US clinic. Eur Radiol 2012; 22: 957-965. 